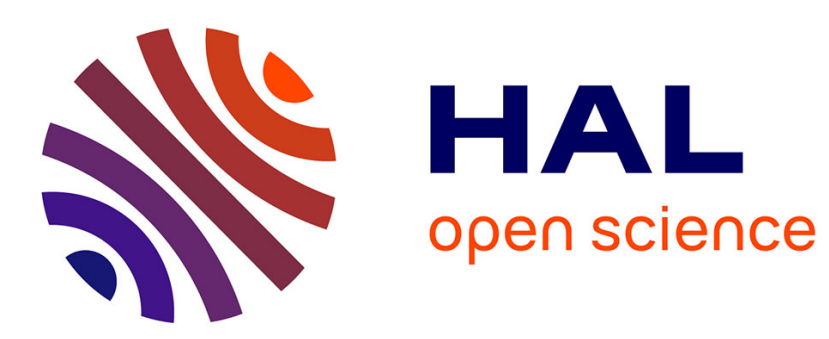

\title{
Guillain-Barré syndrome subtypes related to Campylobacter infection
}

Judith Drenthen, Nobuhiro Yuki, Jan Meulstee, Ellen M Maathuis, Pieter A Doorn Van, Gerhard H Visser, Joleen H Blok, Bart C Jacobs

\section{To cite this version:}

Judith Drenthen, Nobuhiro Yuki, Jan Meulstee, Ellen M Maathuis, Pieter A Doorn Van, et al.. Guillain-Barré syndrome subtypes related to Campylobacter infection. Journal of Neurology, Neurosurgery and Psychiatry, 2011, 82 (3), pp.300. 10.1136/jnnp.2010.226639 . hal-00611250

\section{HAL Id: hal-00611250 \\ https://hal.science/hal-00611250}

Submitted on 26 Jul 2011

HAL is a multi-disciplinary open access archive for the deposit and dissemination of scientific research documents, whether they are published or not. The documents may come from teaching and research institutions in France or abroad, or from public or private research centers.
L'archive ouverte pluridisciplinaire HAL, est destinée au dépôt et à la diffusion de documents scientifiques de niveau recherche, publiés ou non, émanant des établissements d'enseignement et de recherche français ou étrangers, des laboratoires publics ou privés. 


\section{Guillain-Barré syndrome subtypes related to Campylobacter infection}

Judith Drenthen, ${ }^{1,2}$ Nobuhiro Yuki, ${ }^{3}$ Jan Meulstee, ${ }^{4}$ Ellen M. Maathuis, ${ }^{1}$ Pieter A. van Doorn, ${ }^{2}$ Gerhard H. Visser, ${ }^{1}$ Joleen H. Blok, ${ }^{1}$ Bart C. Jacobs ${ }^{2,5}$

${ }^{1}$ Depts. of Clinical Neurophysiology, ${ }^{2}$ Neurology, and ${ }^{5}$ Immunology, Erasmus MC, University Medical Center Rotterdam, The Netherlands. ${ }^{3}$ Depts. of Microbiology and Medicine, National University of Singapore, Singapore. ${ }^{4}$ Dept. of Clinical Neurophysiology, Canisius-Wilhelmina Hospital, Nijmegen, The Netherlands

Corresponding author

Judith Drenthen, Department of Clinical Neurophysiology, Erasmus MC, University Medical Center Rotterdam, P.O. Box 2040, 3000 CA Rotterdam, The Netherlands

Tel +31 107033759, Fax +31 107034621

E-mail: j.drenthen@erasmusmc.nl

Word count

Word count paper: 3313; Word count abstract: 249; Character count title: 67; Number of references: 34; Number of tables: 3; Number of figures: 0

Search terms

Guillain-Barré syndrome, EMG, Cytomegalovirus, Epstein-Barr virus , Campylobacter jejuni 


\section{ABSTRACT}

Background: In Guillain-Barré syndrome (GBS) the diversity in electrophysiological subtypes is unexplained, but may be determined by geographical factors and preceding infections. Acute motor axonal neuropathy (AMAN) is a frequent GBS variant in Japan and one study proposed that in Japan Campylobacter jejuni infections exclusively elicit AMAN. In the Netherlands $C$. jejuni is the predominant type of preceding infection, yet AMAN is rare. This may indicate that not all Dutch GBS patients with $C$. jejuni infections have AMAN. Objective: To determine if GBS patients with a preceding $C$. jejuni infection in the Netherlands exclusively have AMAN.

Methods: Retrospective analysis of preceding infections in relation to serial electrophysiology and clinical data from 123 GBS patients. C. jejuni-related cases were defined as having preceding diarrhea and positive C. jejuni serology. Electrophysiological characteristics in $C$. jejuni-related cases were compared with those in viral-related GBS patients. In addition, eight GBS patients from another cohort with positive stool cultures for C. jejuni were analysed.

Results: Seventeen (14\%) of 123 patients had C. jejuni-related GBS. C. jejuni patients had lower motor and higher sensory action potentials compared to viral-related cases. Nine (53\%) C. jejuni patients had either AMAN or inexcitable nerves. However, three (18\%) patients fulfilled the criteria for acute inflammatory demyelinating polyneuropathy (AIDP). Also, two (25\%) of eight additional patients with a $C$. jejuni-positive stool sample had AIDP.

Conclusion: In the Netherlands $C$. jejuni infections are strongly, but not exclusively associated with axonal GBS. Some patients with these infections fulfil current criteria for demyelination. 


\section{INTRODUCTION}

Guillain-Barré syndrome (GBS) is a post-infectious polyradiculoneuropathy with variable clinical and electrophysiological subtypes. GBS is commonly subdivided into acute inflammatory demyelinating polyneuropathy (AIDP), acute motor axonal neuropathy (AMAN), and acute motor sensory axonal neuropathy (AMSAN). There is a remarkable difference in geographical distribution of these subtypes.[1] The axonal variants abound in Asia, while AIDP is predominant in western countries.[1-3] This geographical variation is unexplained, but may be related to differences in genetic or environmental factors. Previous studies indicated that preceding infections may influence the electrophysiological subtype of GBS.[4-6] More specifically, a recent study proposed that in Japan infections with Campylobacter jejuni are exclusively related to AMAN.[7] The high incidence of $C$. jejuni infections in GBS patients in Japan would explain why axonal variants predominate there.

Patients from western countries most frequently report symptoms of preceding respiratory tract infections. Indeed, serological studies have yielded evidence for infections with cytomegalovirus (CMV), Epstein-Barr virus (EBV) and Mycoplasma pneumoniae.(5) Nevertheless, in these regions $C$. jejuni is the predominant type of infection preceding GBS, that may affect $>30 \%$ of patients. [8, 9] AMAN in western countries is rare $(<10 \%),[3,10]$ however, suggesting that $C$. jejuni in western patients may also induce other GBS subtypes. Here, we defined what subtypes of GBS may occur after $C$. jejuni infection in the Netherlands. $C$. jejuni infections were defined by specific techniques, including those used in Japanese studies, to exclude methodological bias. Patients with $C$. jejuni infections were assessed in serial electrophysiological studies and compared with patients with preceding viral infections. 


\section{PATIENTS AND METHODS}

\section{Patients}

The patients for our study were derived from a cohort of 147 GBS patients who participated in a randomised trial comparing the therapeutic effect of intravenous immunoglobulins (IVIg) versus plasmapheresis.[11] All patients fulfilled the diagnostic criteria for GBS, were admitted to the hospital within two weeks of onset of weakness, and were unable to walk ten meters independently (GBS disability score > 2).[12] During a follow-up of six months, the patients underwent neurological assessments at standardised time points according to a predefined protocol. During these visits, clinical parameters such as sensory abnormalities, cranial nerve involvement, GBS disability scales, and MRC sum scores were determined.[13, 14] Informed consent was obtained from all patients and the study was approved by the local medical ethics committee. Data from serial electrophysiological studies and pretreatment serum samples were available from $123(84 \%)$ of the 147 patients. These patients were not significantly different from the excluded 24 cases regarding baseline and clinical characteristics.

To confirm our findings in patients with positive $C$. jejuni serology in this cohort, we analysed patients with positive $C$. jejuni stool cultures from a second cohort who participated in a trial studying the effect of combined IVIg and methylprednisolone treatment.[15] For this later cohort, stool specimens were available, allowing isolation of $C$. jejuni bacteria directly from these specimens (the gold standard for identification of a recent $C$. jejuni infection). In this second cohort, eight (4\%) of 225 GBS patients were identified with a positive stool culture for C. jejuni. All eight patients of this second cohort fulfilled the diagnostic criteria for GBS, underwent an extensive follow-up, and had an EMG within three weeks after randomisation.[15] 


\section{Electrophysiology}

Electrophysiological findings vary considerably in the acute stage of GBS. This dynamic situation may lead to changes in classification of patients depending on the timing of the EMG. Therefore, in this study, serial EMG studies were performed at three standard time points: within two days of hospital admission, after one week, and after one month.[10] Kuwabara et al. categorized their EMGs based on the time elapsed since the onset of weakness rather than the time of admission.[7] To be able to compare our study with this previous study, we chose to analyse the EMGs that were made in the second week after onset of weakness (median 11 days, 95\% CI 10-12 days) and more than 4 weeks after onset of weakness (median 33 days, 95\% CI 32-38 days).

Motor and sensory nerve conduction studies were performed on the ulnar and median nerves. Stimulation and recordings were performed at standardised sites.[16, 17] Recordings from motor nerves were quantified using peak-to-peak distal and proximal compound motor action potential amplitudes (dCMAP and pCMAP), distal motor latency (DML), motor nerve conduction velocity (mNCV), and F-wave latencies. For sensory nerves, the peak-to-peak sensory nerve action potential (SNAP) amplitude and sensory nerve conduction velocity (sNCV) were measured. Needle EMG was performed in the anterior tibial muscle, abductor pollicis brevis muscle or the abductor digiti minimi muscle to determine the presence of denervation activity (fibrillation potentials and positive sharp waves). In all investigations, the electromyographers were blinded for the results of infection serology. The EMGs were classified as demyelinating, axonal, inexcitable, equivocal, or normal according to the Hadden criteria.[3] :

Normal: no abnormalities in any single nerve. Primary demyelinating: at least one of the following in each of at least two nerves, or at least two of the following in one nerve if all others are inexcitable and dCMAP $>10 \%$ of lower limit of normal (LLN): DML $>110 \%$ 
upper limit of normal (ULN) (120\% if dCMAP < 100\% LLN); mNCV < 90\% LLN (85\% if dCMAP < 50\% LLN); F-wave latency >120\% ULN; Conduction block (CB): CMAP/dCMAP ratio $\leq 50 \%$ (if dCMAP $\geq 20 \%$ LLN). Primary axonal: no demyelinating features (except one feature allowed if dCMAP < 10\% LLN) and dCMAP <80\% LLN in 2 or more nerves. Inexcitable: dCMAP absent in all nerves (or present in 1 nerve with dCAMP $<10 \%$ LLN). Equivocal: does not fit criteria for any other group. Nerves with a distal CMAP (dCMAP) < $10 \%$ LLN did not change the classification.

Reference values were defined previously.[17] The temperature of the recording- and stimulation sites were registered.

\section{Serological studies}

Pre-treatment serum samples were examined for evidence of a recent $C$. jejuni infection using tests from both Japanese and Dutch laboratories (Dokkyo Medical University in Tochigi, Japan, and SSDZ, Delft, The Netherlands).[18, 19] Both tests were based on an ELISA, and have been described in detail previously.[20, 21] In the Japanese laboratory, serological evidence of a recent $C$. jejuni infection was defined as anti- $C$. jejuni $\operatorname{IgG}$ titres of 2,000 or more; in the Dutch laboratory as the presence of anti-C. jejuni $\operatorname{IgM}$ or IgA antibodies at higher levels than an internal standard.[8] Differences between the Japanese and Dutch tests were described previously. [19] Both tests were used in the current study to exclude the possibility that the serology method introduced a bias. Patients were regarded $C$. jejuni-positive if they: (1) suffered from diarrhea in the three weeks preceding the onset of weakness, and (2) were tested positive for $C$. jejuni serology in either one of the two independent laboratories. The $C$. jejuni-related patients were compared with a group of GBS patients from the same trial cohort who had a positive serology for a recent infection with CMV or EBV according to previously defined criteria.[8] Furthermore, the pretreatment serum samples were tested for $\operatorname{IgM}$ and $\operatorname{IgG}$ 
antibody reactivity against GM1 and GD1a (in The Netherlands), and against GM1, GM1b, and GalNAc-GD1a (in Japan) using ELISA as previously described.[22, 23]

\section{Statistical analysis}

Normally distributed data were analysed using the two-sided $t$-test for independent samples. Ordinal data and not normally distributed data were analysed using the two-sided Wilcoxon rank sum score test. The $\chi^{2}$ test or Fisher exact test was used for categorical data depending on sample size. Data are presented as means and standard deviations (SD) or as medians and $95 \%$ confidence intervals $(95 \% \mathrm{CI})$. For categorical variables, frequencies and percentages are given. All calculations were performed using SPSS 15.0 (SPSS Inc, Chicago, IL). A $p$-value of $<0.05$ was considered to be statistically significant. 


\section{RESULTS}

\section{Comparison of patients with $C$. jejuni- versus CMV/EBV-related GBS}

The electrophysiology of the patients with positive $C$. jejuni serology was compared with that of patients with a positive CMV or EBV serology. Seventeen (14\%) of the cohort of 123 GBS patients met our strict criteria for a recent infection with $C$. jejuni (diarrhea and positive $C$. jejuni serology). In the same cohort of patients 24 (20\%) had a positive serology for either CMV or EBV. The electrophysiological characteristics of the CMV-and EBV-related patients were very similar; therefore, both groups were combined for further analysis. The mean age of the $C$. jejuni-related patients ( $56 \pm 17$ years) was significantly higher than of the CMV/EBV-related patients $(36 \pm 15$ years $)(p<0.001)$. Sensory deficits were less frequent in the $C$. jejuni-related patients $(41 \%)$ compared to the CMV/EBV-related patients $(78 \%)(p=$ 0.02). The median MRC sum score after six months was lower in the C. jejuni-related patients (44 with 95\% CI 31-58) than in the CMV/EBV-related patients (58 with 95\% CI 52-60) ( $p=$ 0.004). The median GBS disability score at six months was significantly higher for the $C$. jejuni-related patients (3 with 95\% CI 1-4) compared to the CMV/EBV-related patients (1 with $95 \%$ CI 1-2) $(p=0.02)$, indicating a poorer outcome. Anti-ganglioside antibodies were present in $88 \%$ of the $C$. jejuni-related patients and in $4 \%$ of the CMV/EBV-related patients $(p<0.001)$

The two groups differed considerably with respect to electrophysiological features and subtypes, as shown in Table 1 . The $C$. jejuni-related patients had significantly lower CMAP amplitudes and lower DMLs, but higher SNAP amplitudes. These differences were found in both the ulnar and median nerves, although the differences in the ulnar nerves were more pronounced.

The frequency of denervation activity (fibrillation potentials and/or positive sharp waves) in the acute phase and at 4 weeks did not differ significantly between the two groups. Within 2 
weeks, $20 \%$ of the $C$. jejuni patients and $21 \%$ of the CMV/EBV patients showed denervation activity (p=0.95). After 4 weeks, $69 \%$ of the $C$. jejuni patients versus $56 \%$ of the CMV/EBV patients showed active denervation $(\mathrm{p}=0.44)$.

In five patients from the CMV/EBV-related group, an EMG was performed only within two weeks and not after four weeks. At four weeks, the majority of EMGs in the $C$. jejuni-related group were classified as the axonal type or as inexcitable, but two patients (12\%) had a demyelinating type of EMG. By contrast, at that time $85 \%$ of the EMGs of the CMV/EBVrelated patients were classified as demyelinating.

The distal temperature during all EMG registrations was not decreased, except for two patients. Patient number 11 in the $C$. jejuni-related group had a temperature at the wrist of 29 ${ }^{\circ} \mathrm{C}$ and was classified as axonal in the first EMG and as equivocal in the second EMG. One patient from the CMV/EBV-related group had a temperature at the wrist of $29^{\circ} \mathrm{C}$ and had normal electrophysiology at both time points. 
Table 1 Electrophysiological findings in the ulnar nerve of GBS patients with positive $\boldsymbol{C}$. jejuni serology versus GBS patients with positive CMV/EBV serology.

\begin{tabular}{|c|c|c|c|}
\hline & C. jejuni $(\mathrm{n}=17)$ & CMV/EBV $(\mathrm{n}=24) *$ & $p$-value \\
\hline \multicolumn{4}{|l|}{ EMG < 2weeks } \\
\hline Ulnar CMAP (mV) & $0.3(0.0-3.0)$ & $4.0(1.9-5.0)$ & 0.01 \\
\hline Ulnar SNAP $(\mu \mathrm{V})$ & $10.0(3.6-18.5)$ & $1.9(0-8.0)$ & 0.02 \\
\hline Ulnar mNCV (m/s) & $52(46-60)$ & $47(42-53)$ & 0.08 \\
\hline Ulnar DML (ms) & $3.4(2.7-4.6)$ & $4.6(3.9-5.7)$ & 0.04 \\
\hline Classification (\%) & & & $<0.001$ \\
\hline Demyelinating & $2(12 \%)$ & $21(88 \%)$ & \\
\hline Axonal & $5(29 \%)$ & $1(4 \%)$ & \\
\hline Inexcitable & $4(24 \%)$ & $0(0 \%)$ & \\
\hline Equivocal & $6(35 \%)$ & $1(4 \%)$ & \\
\hline Normal & $0(0 \%)$ & $1(4 \%)$ & \\
\hline \multicolumn{4}{|l|}{ EMG > 4weeks } \\
\hline Ulnar CMAP (mV) & $0.4(0.0-3.5)$ & $5.4(2.0-10.0)$ & 0.01 \\
\hline Ulnar SNAP $(\mu \mathrm{V})$ & $16.5(4.0-26.0)$ & $2.5(0.0-6.3)$ & 0.01 \\
\hline Ulnar mNCV (m/s) & $49(37-59)$ & $44(39-50)$ & 0.12 \\
\hline Ulnar DML (ms) & $3.6(2.8-5.0)$ & $5.6(4.3-7.1)$ & 0.003 \\
\hline Classification (\%) & & & $<0.001$ \\
\hline Demyelinating & $2(12 \%)$ & $16(85 \%)$ & \\
\hline Axonal & $5(29 \%)$ & $0(0 \%)$ & \\
\hline Inexcitable & $4(24 \%)$ & $1(5 \%)$ & \\
\hline Equivocal & $6(35 \%)$ & $1(5 \%)$ & \\
\hline Normal & $0(0 \%)$ & $1(5 \%)$ & \\
\hline
\end{tabular}

The values for the ulnar CMAP, SNAP, DML and mNCV are presented as medians (and 95\% CI between brackets). EMGs were made within two weeks (median 11 days) and after 4 weeks (median 33 days) after weakness onset. Classifications are presented as numbers (and percentages between brackets). * EMG at > 4 weeks was missing in five $(21 \%)$ of $24 \mathrm{CMV}$ EBV-related GBS patients. 
Classification criteria according to Hadden et al.[3]:

Normal: no abnormalities in any single nerve.

Demyelinating: one of the following in two nerves (or two of the following in one nerve if all others are inexcitable and dCMAP > 10\% LLN): DML > 110\% ULN $(120 \%$ if dCMAP < $100 \%$ LLN); mNCV < 90\% LLN (85\% if dCMAP < 50\% LLN); F-wave latency >120\% ULN; Conduction block (CB): CMAP/dCMAP ratio $\leq 50 \%$ (if dCMAP $\geq 20 \%$ LLN). Axonal: no demyelinating features (except one feature if dCMAP $<10 \%$ LLN) and dCMAP $<80 \%$ LLN in 2 or more nerves.

Inexcitable: dCMAP absent in all nerves (or present in 1 nerve with dCAMP $<10 \%$ LLN). , Equivocal: does not fit criteria for any other group.

Nerves with a distal CMAP $($ dCMAP) $<10 \%$ LLN did not change the classification.

\section{Classification of patients with positive $C$. jejuni serology}

Nine (53\%) of the 17 patients with positive C. jejuni serology tested positive in both the Dutch and the Japanese assay; the other eight patients tested positive in the Dutch assay only. These two subgroups were indistinguishable in terms of clinical and electrophysiological characteristics and, therefore, were combined for further analysis. Table 2 provides an overview of the electrophysiological and serological data for all C. jejuni-serology positive patients.

Three of these 17 patients with positive $C$. jejuni serology fulfilled the electrophysiological criteria of Hadden for demyelination at one or both time points (Table 3). Four weeks after the onset of weakness, when most patients have reached a stable situation, two of these three patients fulfilled the criteria for demyelination. The first patient (study number 6) also had high titers of IgG antibodies to GM1 in the Japanese and Dutch assays, normal SNAPs, and a purely motor form of GBS. Needle EMG did not show any signs of axonal damage in the 
form of fibrillation potentials and/or positive sharp waves. The second patient (study number 15) had high titers of IgG antibodies to GM1 in the Dutch assay and of GM1, GM1b and GalNAc-GD1a in the Japanese assay. This patient had abnormal SNAPs. Needle EMG also showed signs of axonal involvement (fibrillation and positive sharp waves). The third patient (study number 16), tested negative for anti-ganglioside antibodies, had abnormal SNAPs, and no denervation potentials in needle EMG.

\section{Classification of patients with positive $C$. jejuni-stool culture}

Two $(25 \%)$ of the eight patients from the second cohort with a stool sample that was positive for C. jejuni fulfilled the demyelination criteria (Table 3). Patient A was a 63-year-old male who was admitted with a progressive burning sensation and paresthesias in hands and feet and, one day later, progressive muscle weakness. His EMG was performed 19 days after weakness onset and showed prolonged DMLs in combination with low NCVs, a conduction block, and absent SNAPs. Needle EMG showed no denervation activity. He had a positive serology for IgG anti-GM1 antibodies. Patient B was a 36-year-old female, with pronounced muscle weakness and mild sensory disturbance. Her EMG was performed 12 days after weakness onset and showed prolonged DMLs in median, ulnar and peroneal nerve, an abnormal mNCV in the median nerve, and abnormal SNAPs of median and ulnar nerve. Needle EMG showed no denervation activity. No serum antibodies to gangliosides were found. 
Table 2 Electrophysiological classification of GBS patients with diarrhea and positive $\boldsymbol{C}$.

jejuni serology.

\begin{tabular}{|c|c|c|c|c|c|c|}
\hline \multirow[t]{2}{*}{$\mathrm{Nr}$} & \multicolumn{2}{|c|}{ C. jejuni serology } & \multicolumn{2}{|c|}{$\begin{array}{l}\text { Anti-ganglioside } \\
\text { antibodies }\end{array}$} & \multicolumn{2}{|c|}{ EMG classification } \\
\hline & Dutch & $\begin{array}{c}\text { Japa- } \\
\text { nese }\end{array}$ & Dutch & Japanese & $<2$ weeks & $>4$ weeks \\
\hline 1 & + & + & GM1 & GM1 & Axonal & Axonal \\
\hline 2 & + & + & GM1 & $\begin{array}{l}\text { GM1, GM1b, } \\
\text { GalNAc-GD1a }\end{array}$ & Axonal & Axonal \\
\hline 3 & + & + & - & $\begin{array}{c}\text { GM1b, } \\
\text { GalNAc-GD1a }\end{array}$ & Axonal & Equivocal \\
\hline 4 & + & + & $\begin{array}{l}\text { GM1, } \\
\text { GD1a }\end{array}$ & $\begin{array}{l}\text { GM1, GM1b, } \\
\text { GalNAc-GD1a }\end{array}$ & Equivocal & Axonal \\
\hline 5 & + & + & GD1a & $\begin{array}{l}\text { GM1, GM1b, } \\
\text { GalNAc-GD1a }\end{array}$ & Equivocal & Equivocal \\
\hline 6 & + & + & GM1 & GM1, GM1b & Equivocal & Demyelinating \\
\hline 7 & + & + & GM1 & GM1, GM1b & Inexcitable & Inexcitable \\
\hline 8 & + & + & GM1 & $\begin{array}{l}\text { GM1, GM1b, } \\
\text { GalNAc-GD1a }\end{array}$ & Inexcitable & Inexcitable \\
\hline 9 & + & + & GM1 & GM1, GM1b & Inexcitable & Inexcitable \\
\hline 10 & + & - & - & GalNAc-GD1a & Axonal & Axonal \\
\hline 11 & + & - & GM1 & $\begin{array}{c}\text { GM1b, } \\
\text { GalNAc-GD1a }\end{array}$ & Axonal & Equivocal \\
\hline 12 & + & - & - & $\begin{array}{c}\text { GM1b, } \\
\text { GalNAc-GD1a }\end{array}$ & Equivocal & Axonal \\
\hline 13 & + & - & - & - & Equivocal & Equivocal \\
\hline 14 & + & - & - & GM1b & Equivocal & Equivocal \\
\hline 15 & + & - & GM1 & $\begin{array}{l}\text { GM1, GM1b, } \\
\text { GalNAc-GD1a }\end{array}$ & Demyelinating & Demyelinating \\
\hline 16 & + & - & - & - & Demyelinating & Equivocal \\
\hline 17 & + & - & $\begin{array}{l}\text { GM1, } \\
\text { GD1b }\end{array}$ & Not tested & Inexcitable & Inexcitable \\
\hline
\end{tabular}


C. jejuni serology and anti-ganglioside antibodies were determined in two independent (Dutch and Japanese) laboratories. EMGs were made within 2 weeks (median 11 days) and after 4 weeks (median 33 days) after weakness.

Classification criteria according to Hadden et al. [3]:

Demyelinating: one of the following two nerves (or two of the following in one nerve if all others are inexcitable and dCMAP > 10\% LLN): DML > 110\% ULN $(120 \%$ if dCMAP < $100 \% \mathrm{LLN}) ; \mathrm{mNCV}<90 \%$ LLN (85\% if dCMAP < 50\% LLN); F-wave latency $>120 \%$ ULN; Conduction block (CB): CMAP/dCMAP ratio $\leq 50 \%$ (if dCMAP $\geq 20 \%$ LLN). Axonal: no demyelinating features (one feature allowed if dCMAP < 10\% LLN) and dCMAP $<80 \%$ LLN in 2 or more nerves.

Inexcitable: dCMAP absent in all nerves (or present in 1 nerve with dCAMP $<10 \%$ LLN). Equivocal: does not fit criteria for any other group. 
Table 3 Patients positive for $C$. jejuni-serology or -culture fulfilling the criteria for a demyelinating form of GBS.

$$
\text { C. jejuni-serology positive }
$$

C. jejuni-stool culture positive

$\begin{array}{llllll}\text { Patient } & 6 & 15 & 16 & \text { A } & \text { B }\end{array}$

No.

\begin{tabular}{|c|c|c|c|c|c|c|c|c|}
\hline $\begin{array}{l}\text { Time } \\
\text { point }\end{array}$ & $<2 \mathrm{wks}$ & $>4 \mathrm{wks}$ & $<2 \mathrm{wks}$ & $>4$ wks & $<2 w k s$ & $>4 \mathrm{wks}$ & 19 days & 12 days \\
\hline \multicolumn{9}{|c|}{ Median nerve } \\
\hline $\mathrm{mNCV}$ & 50 & 38 & $\S$ & $\S$ & 59 & 53 & 38 & 37 \\
\hline$(\mathrm{m} / \mathrm{s})$ & $(98 \%)$ & $(75 \%)$ & & & $(111 \%)$ & $(100 \%)$ & $(81 \%)$ & $(70 \%)$ \\
\hline DML & 3.2 & 3.7 & $\S$ & $\S$ & 11.2 & 7.6 & 4.8 & 6.0 \\
\hline (ms) & $(73 \%)$ & $(84 \%)$ & & & $(255 \%)$ & $(173 \%)$ & $(102 \%)$ & $(136 \%)$ \\
\hline F-wave & - & - & $\S$ & $\S$ & 54.9 & 37.3 & - & 39.5 \\
\hline (ms) & & & & & $(164 \%)$ & $(113 \%)$ & & $(128 \%)$ \\
\hline abnormal & - & - & + & + & + & + & + & + \\
\hline \multicolumn{9}{|l|}{ SNAP } \\
\hline $\mathrm{CB}$ & - & - & - & - & - & - & - & - \\
\hline \multicolumn{9}{|c|}{ Ulnar nerve } \\
\hline $\mathrm{mNCV}$ & 58 & 37 & 43 & 28 & 64 & 56 & 43 & 54 \\
\hline$(\mathrm{m} / \mathrm{s})$ & $(112 \%)$ & $(71 \%)$ & $(83 \%)$ & $(54 \%)$ & $(123 \%)$ & $(108 \%)$ & $(83 \%)$ & $(102 \%)$ \\
\hline DML & 3.3 & 5.0 & 4.6 & 3.6 & 4.3 & 4.0 & 3.3 & 4.1 \\
\hline (ms) & $(89 \%)$ & $(135 \%)$ & $(124 \%)$ & $(97 \%)$ & $(116 \%)$ & $(108 \%)$ & $(89 \%)$ & $(111 \%)$ \\
\hline F-wave & - & - & - & 44 & - & 36.1 & 39.7 & 33.9 \\
\hline (ms) & & & & $(130 \%)$ & & $(110 \%)$ & $(117 \%)$ & $(112 \%)$ \\
\hline abnormal & - & - & + & + & + & + & + & - \\
\hline \multicolumn{9}{|l|}{ SNAP } \\
\hline $\mathrm{CB}$ & - & - & + & + & - & - & - & - \\
\hline
\end{tabular}

Electrophysiological findings in patients with positive C. jejuni serology (patient 6, 15 and 16) or stool culture (patient A and B). Absolute values of nerve conduction velocity (NCV), distal motor latency (DML) and F-wave latency are shown. Between brackets are given the 
percentages of lower limits of normal (LLN) for $\mathrm{mNCV}$ and percentages of the upper limit of normal (ULN) for DML and F-waves. Normal values (and related LLN and ULN) depend on sex, age, and length. Abnormal SNAP amplitude and presence of conduction blocks (CB) are indicated by '-' and '+'. At the time points in bold face (second row), the EMGs were classified as demyelinating. The numbers in bold face represent the abnormalities in the "demyelinating range". [3] Nerves with a distal CMAP (dCMAP) $<10 \%$ did not change the classification.

$\S=\mathrm{dCMAP}<10 \% \mathrm{LLN}$ 


\section{DISCUSSION}

The current study confirmed the previous study of Kuwabara et al. that in GBS patients, a preceding infection with $C$. jejuni is frequently followed by AMAN, even in western countries where this axonal subtype is relatively rare.[3] The majority of Dutch patients with a preceding C. jejuni infection developed a variant with inexcitable nerves or features of axonal degeneration in serial EMG studies. In contrast, Japanese and Dutch patients with a preceding EBV or CMV infection rarely develop AMAN, indicating that preceding infections influence the electrophysiological subtype of GBS. However, even when very strict clinical and serological criteria for a recent infection with $C$. jejuni were applied, three (18\%) of 17 GBS patients with a $C$. jejuni fulfilled the electrophysiological criteria for AIDP. Furthermore, in another cohort, $C$. jejuni was cultured from the stools of two patients with a typical demyelinating form of GBS. These findings demonstrate that at least in Dutch patients, $C$. jejuni infections do not exclusively elicit the AMAN variant of GBS. This finding is in accordance with the relatively high frequency of both $C$. jejuni infections and AIDP in the Netherlands.

To be able to clarify which GBS subtypes can be elicited by a $C$. jejuni infection, our first priority was to prevent the inclusion of false-positive $C$. jejuni cases. For that reason, an extensively validated serological assay was used, which has a sensitivity of $96 \%$ and a specificity of $93 \%$ for detecting a recent $C$. jejuni infection in patients with GBS.[18] To further increase the specificity, we added the criterium of presence of preceding diarrhea. This combination of strict criteria explains the low frequency of $C$. jejuni-related cases compared to previous studies.[5, 22, 24] The specificity of the $C$. jejuni serology may differ among assays.[2, 19] To make our results as comparable as possible to previous studies from Japan,[7] serological testing for C. jejuni was performed in the Netherlands as well as in Japan. The findings that anti-ganglioside antibodies were almost exclusively found in the 
patients with positive $C$. jejuni serology, lend further support to a high specificity of the assays. Furthermore, in two GBS patients with a positive stool culture for $C$. jejuni, the gold standard for identifying a recent infection, demyelinating forms were identified. Taken together, it is highly unlikely that the observed coincidence of $C$. jejuni infection and AIDP in five out of the total 25 C. jejuni-positive patients can be explained by false-positive cases of C. jejuni.

The second issue that needs to be addressed in this context is whether the electrophysiological features observed in these five patients can be attributed rightly to the process of demyelination. In the current study we have used the criteria of Hadden et al.[3] These criteria were developed in Caucasian GBS patients, but are similar to the criteria of Ho et al., [2] that were used in the previous study from Japan.[7] All patients in the current study that were classified as AIDP with the Hadden's criteria, including the two C. jejuni-culture positive patients, also fulfilled these criteria for AIDP of Ho et al. Over the years, multiple sets of electrophysiological (research) criteria have been developed to identify demyelination in GBS patients.[2, 3, 10, 25-27] No set is universally accepted, however, and no comparative studies with nerve biopsies are available for GBS including the current study. Furthermore, a few studies have shown the existence of conduction blocks, conduction slowing, and increased DMLs in AMAN patients.[28, 29] These phenomena were presumably caused by alteration of the resting membrane potential and sodium channel inactivation. Along the same lines, it should be noted that three of the five $C$. jejuni-positive patients with AIDP in our study had high titres of serum IgG antibodies to GM1 or other gangliosides. Only one of these patients also had signs of axonal involvement in the form of fibrillation potentials and positive sharp waves on EMG. Usually, these antibodies are strongly associated with axonal degeneration.[22, 30] Possibly, these findings point to the existence of an as yet unrecognized subtype, which merges axonal and demyelinating characteristics on EMG. Within the limits 
of the currently available criteria (whether those of Hadden, Ho or others), however, the EMGs of the five patients unmistakably show primary demyelinating features. Moreover, the majority had marked sensory abnormalities on the EMG, which are very uncommon in AMAN.[29]

The current study is based on the EMGs of median and ulnar nerves, the only two motor nerves that were studied in this patient cohort systematically. In the study of Kuwabara et al, four motor nerves were examined. [7]. The current criteria require the presence of a demyelinating feature in at least two nerves, which enabled us to classify patients with AIDP. Performing EMG in a third (or fourth) nerve will only increase the sensitivity to detect demyelination.

A third potential confounder for the subtyping of GBS patients is the timing of the EMG, since the electrophysiological findings vary considerably in the acute stage of GBS.[26] This dynamic situation may lead to changes in the classification of patients during follow-up, as was found in our study as well as that of Japan.[7] Four weeks after weakness onset, the situation may be expected to have stabilized. Even at that time, some of our patients fulfilled the electrophysiological criteria for demyelination. In this context, it should also be noted that none of our $C$. jejuni patients with a demyelinating EMG had the previously reported quickly reversible conduction blocks and increased DMLs that normalize within two weeks after weakness onset.[7]

More extensive studies are required to establish the relations between the geographical and ethnical origin of the patient, the type of preceding infections, and the subsequent electrophysiological and clinical phenotype. If the patient's origin indeed influences the phenotype of GBS after infection with $C$. jejuni, this raises the question which factors could explain such an association. Previous studies showed that the type of $C$. jejuni strain and related ganglioside mimicry or additional virulence factors highly influence the subsequent 
neurological deficits.[31] Possibly, these characteristics vary between $C$. jejuni strains derived from different geographical areas. In addition to these pathogen factors, host factors may codetermine the phenotype by influencing the specificity and type of immune response triggered by a preceding $C$. jejuni infection. Alternatively, the antigenic make-up of the peripheral nervous system or accessibility of these targets to the immune system may depend on the origin of the host. Various genetic factors are associated with disease occurrence and severity.[32-34]

Extensive international collaboration and comparisons of large and standardized cohorts of GBS patients from various geographical and ethnical origins are required to resolve these issues. In addition, the current classification into demyelinating and axonal subtypes based on EMG characteristics may be too crude to account for the diversity in clinical, serological, and electrophysiological findings. A more refined classification of patients may lead to more targeted and individualized therapies. 


\section{ACKNOWLEDGEMENTS}

We would like to thank the members of the Dutch GBS Study Group for their participation in the study and gratefully acknowledge financial support from the Prinses Beatrix Fonds (research grant WAR06-215).

\section{COMPETING INTERESTS}

Joleen Blok and Bart C. Jacobs received a research grant from the Princess Beatrix Fund (WAR06-215)

Judith Drenthen has nothing to disclose.

Nobuhiro Yuki has nothing to disclose.

Jan Meulstee has nothing to disclose.

Ellen M. Maathuis has nothing to disclose.

Pieter A. van Doorn has nothing to disclose.

Gerhard H. Visser has nothing to disclose.

Joleen H. Blok has nothing to disclose.

Bart C. Jacobs has nothing to disclose.

\section{COPYRIGHT LICENCE STATEMENT}

The Corresponding Author has the right to grant on behalf of all authors and does grant on behalf of all authors, an exclusive licence on a worldwide basis to the BMJ Group and coowners or contracting owning societies (where published by the BMJ Group on their behalf), and its Licensees to permit this article (if accepted) to be published in Journal of Neurology, Neurosurgery \& Psychiatry and any other BMJ Group products and to exploit all subsidiary rights, as set out in our licence. 


\section{REFERENCE LIST}

1. K. Ogawara, S. Kuwabara, M. Mori, et al. Axonal Guillain-Barre syndrome: relation to anti-ganglioside antibodies and Campylobacter jejuni infection in Japan. Ann Neurol 2000;48:624-631.

2. T. W. Ho, B. Mishu, C. Y. Li, et al. Guillain-Barre syndrome in northern China. Relationship to Campylobacter jejuni infection and anti-glycolipid antibodies. Brain 1995;118 ( Pt 3):597-605.

3. R. D. Hadden, D. R. Cornblath, R. A. Hughes, et al. Electrophysiological classification of Guillain-Barre syndrome: clinical associations and outcome. Plasma Exchange/Sandoglobulin Guillain-Barre Syndrome Trial Group. Ann Neurol 1998;44:780788.

4. C. W. Ang, M. Koga, B. C. Jacobs, et al. Differential immune response to gangliosides in Guillain-Barre syndrome patients from Japan and The Netherlands. J Neuroimmunol 2001;121:83-87.

5. R. D. Hadden, H. Karch, H. P. Hartung, et al. Preceding infections, immune factors, and outcome in Guillain-Barre syndrome. Neurology 2001;56:758-765.

6. L. H. Visser, F. G. van der Meche, J. Meulstee, et al. Cytomegalovirus infection and Guillain-Barre syndrome: the clinical, electrophysiologic, and prognostic features. Dutch Guillain-Barre Study Group. Neurology 1996;47:668-673.

7. S. Kuwabara, K. Ogawara, S. Misawa, et al. Does Campylobacter jejuni infection elicit "demyelinating" Guillain-Barre syndrome? Neurology 2004;63:529-533.

8. B. C. Jacobs, P. H. Rothbarth, F. G. van der Meche, et al. The spectrum of antecedent infections in Guillain-Barre syndrome: a case-control study. Neurology 1998;51:1110-1115. 9. R. A. Hughes and D. R. Cornblath. Guillain-Barre syndrome. Lancet 2005;366:16531666. 
10. J. Meulstee and F. G. van der Meche. Electrodiagnostic criteria for polyneuropathy and demyelination: application in 135 patients with Guillain-Barre syndrome. Dutch GuillainBarre Study Group. J Neurol Neurosurg Psychiatry 1995;59:482-486.

11. F. G. van der Meche and P. I. Schmitz. A randomized trial comparing intravenous immune globulin and plasma exchange in Guillain-Barre syndrome. Dutch Guillain-Barre Study Group. N Engl J Med 1992;326:1123-1129.

12. A. K. Asbury and D. R. Cornblath. Assessment of current diagnostic criteria for Guillain-Barre syndrome. Ann Neurol 1990;27 Suppl:S21-24.

13. R. P. Kleyweg, F. G. van der Meche and P. I. Schmitz. Interobserver agreement in the assessment of muscle strength and functional abilities in Guillain-Barre syndrome. Muscle Nerve 1991;14:1103-1109.

14. I. S. Merkies, P. I. Schmitz, F. G. van der Meche, et al. Connecting impairment, disability, and handicap in immune mediated polyneuropathies. J Neurol Neurosurg Psychiatry 2003;74:99-104.

15. R. van Koningsveld, P. I. Schmitz, F. G. Meche, et al. Effect of methylprednisolone when added to standard treatment with intravenous immunoglobulin for Guillain-Barre syndrome: randomised trial. Lancet 2004;363:192-196.

16. F. G. van der Meche, J. Meulstee, M. Vermeulen, et al. Patterns of conduction failure in the Guillain-Barre syndrome. Brain 1988;111 ( Pt 2):405-416.

17. R. M. Buschbacher and N. D. Prahlow. Manual of nerve conduction studies, second ed: New York: Demos medical publishing, 2006.

18. C. W. Ang, K. Krogfelt, P. Herbrink, et al. Validation of an ELISA for the diagnosis of recent Campylobacter infections in Guillain-Barre and reactive arthritis patients. Clin Microbiol Infect 2007;13:915-922. 
19. M. Koga, C. W. Ang, N. Yuki, et al. Comparative study of preceding Campylobacter jejuni infection in Guillain-Barre syndrome in Japan and The Netherlands. J Neurol Neurosurg Psychiatry 2001;70:693-695.

20. M. Koga, N. Yuki, M. Takahashi, et al. Close association of IgA anti-ganglioside antibodies with antecedent Campylobacter jejuni infection in Guillain-Barre and Fisher's syndromes. J Neuroimmunol 1998;81:138-143.

21. P. Herbrink, H. A. van den Munckhof, M. Bumkens, et al. Human serum antibody response in Campylobacter jejuni enteritis as measured by enzyme-linked immunosorbent assay. Eur J Clin Microbiol Infect Dis 1988;7:388-393.

22. B. C. Jacobs, P. A. van Doorn, P. I. Schmitz, et al. Campylobacter jejuni infections and anti-GM1 antibodies in Guillain-Barre syndrome. Ann Neurol 1996;40:181-187.

23. B. C. Jacobs, H. Endtz, F. G. van der Meche, et al. Serum anti-GQ1b IgG antibodies recognize surface epitopes on Campylobacter jejuni from patients with Miller Fisher syndrome. Ann Neurol 1995;37:260-264.

24. J. H. Rees, S. E. Soudain, N. A. Gregson, et al. Campylobacter jejuni infection and Guillain-Barre syndrome. N Engl J Med 1995;333:1374-1379.

25. J. W. Albers, P. D. Donofrio and T. K. McGonagle. Sequential electrodiagnostic abnormalities in acute inflammatory demyelinating polyradiculoneuropathy. Muscle Nerve $1985 ; 8: 528-539$.

26. D. R. Cornblath. Electrophysiology in Guillain-Barre syndrome. Ann Neurol 1990;27 Suppl:S17-20.

27. The Italian Guillain-Barre Study Group. The prognosis and main prognostic indicators of Guillain-Barre syndrome. A multicentre prospective study of 297 patients. . Brain 1996;119 ( Pt 6):2053-2061. 
28. M. Capasso, C. M. Caporale, F. Pomilio, et al. Acute motor conduction block neuropathy Another Guillain-Barre syndrome variant. Neurology 2003;61:617-622.

29. A. Uncini and N. Yuki. Electrophysiologic and immunopathologic correlates in Guillain-Barre syndrome subtypes. Expert Rev Neurother 2009;9:869-884.

30. N. Yuki, H. Yoshino, S. Sato, et al. Acute axonal polyneuropathy associated with antiGM1 antibodies following Campylobacter enteritis. Neurology 1990;40:1900-1902.

31. I. Nachamkin, B. M. Allos and T. Ho. Campylobacter species and Guillain-Barre syndrome. Clin Microbiol Rev 1998;11:555-567.

32. N. Yuki, S. Sato, T. Itoh, et al. HLA-B35 and acute axonal polyneuropathy following Campylobacter infection. Neurology 1991;41:1561-1563.

33. N. Yuki, M. Takahashi, Y. Tagawa, et al. Association of Campylobacter jejuni serotype with antiganglioside antibody in Guillain-Barre syndrome and Fisher's syndrome. Ann Neurol 1997;42:28-33.

34. K. Geleijns, A. Roos, J. J. Houwing-Duistermaat, et al. Mannose-binding lectin contributes to the severity of Guillain-Barre syndrome. J Immunol 2006;177:4211-4217. 
The material in this paper has been presented in poster format at the AANEM 55th Annual Meeting in Providence, RI, September 16-20, 2008 and at the Peripheral Nerve Society meeting in Würzburg, Germany, July 4-8, 2009. 